\title{
Diseño y artesanado: una mirada contemporánea
}

Design and craftsmanship: a contemporary view

Pedro Arturo Martinez-Osorio
Universidade Estadual Paulista, UNESP. Bauru (Brasil)
Luis Carlos Paschoarelli

Universidade Estadual Paulista, UNESP. Bauru (Brasil)

Paula Da-Cruz-Landim

Universidade Estadual Paulista, UNESP. Bauru (Brasil)

\author{
Pedro Arturo Martinez-Osorio \\ Arquitecto, Universidad Católica de Colombia. Bogotá, D. C., Colombia \\ Magíster en educación, Universidad Simón Bolívar. Barranquilla, Colombia. \\ Doctor en Design, Universidade Estadual Paulista (UNESP). Bauru, Brasil. \\ https://scholar.google.es/citations?user =PjlmUTsAAAAJ\&hl=es\&oi=ao \\ (i) https://orcid.org/0000-0002-9024-0918 \\ pedro.martinez@cecar.edu.co \\ Luis Carlos Paschoarelli \\ Diseñador industrial, Universidade Estadual Paulista UNESP. Bauru, Brasil. \\ Master en Design Universidade Estadual Paulista, UNESP. Bauru-Brasil. \\ Doctor en Engenharia de Produção, UFS. São Carlos, Brasil. \\ Posdoctorado en ergonomía, Universidad de Lisboa. Lisboa, Portugal. \\ - https://scholar.google.es/citations?user=C8bITFAAAAAJ\&hl=es\&oi=ao \\ (iD https://orcid.org/0000-0002-4685-0508 \\ paschoarelli@faac.unesp.br \\ Paula Da-Cruz-Landim \\ Arquitecta, Faculdade de Arquitetura e Urbanismo da Universidade de São Paulo \\ (FAU-USP) (1987) \\ Magíster en geografia, Instituto de Geociências e Ciências Exatas da Universidade Esta- \\ dual Paulista (UNESP). Rio Claro. \\ Doctorado en arquitectura y urbanismo, Faculdade de Arquitetura e Urbanismo da \\ Universidade de São Paulo (FAU-USP). \\ Estágio de pós-doutorado na Universidade de Arte e Design de Helsinque na Finlândia. \\ https://scholar.google.es/citations?user = gwLh6VcAAAA|\&hl=es\&oi=ao \\ (D) https://orcid.org/0000-0002-1510-7738 \\ paula@faac.unesp.br
}

\section{Resumen}

El escaso reconocimiento de la identidad y de la estética presentes en el artesanado y el uso de la caña flecha (Gynerium sagittatum) por parte de las culturas indígenas zenúes plantea la necesidad de indagar sobre cómo entender el diálogo entre el diseño, el artesanado y los procesos de producción industrial orientados hacia la innovación de procesos y productos en un contexto de sustentabilidad. Mediante una revisión bibliográfica organizada en dos fases (la primera, consistente en la búsqueda de artículos científicos y libros, y la segunda, en el análisis de estos), se pudo clasificar la información en dos categorías: I) Design, Handcraft y nuevas tendencias y 2) Design y Decolonization. Como resultado, se muestran aspectos críticos en la relación entre diseño, artesanado y conocimiento indígena. En esta búsqueda se discute sobre el potencial que, desde el diseño, hace posible generar procesos de innovación; además, se presenta el diseño contemporáneo como una disciplina de síntesis que responde a un enfoque culturalista o esencialista del diseño con grandes capacidades de empoderamiento y emancipación en contextos periféricos. Todo ello permitió cuestionar cuáles metodologías permiten acercarse a la comprensión del legado de las culturas y los procesos de artesanado.

Palabras clave: artesanía; diseño centrado en el sujeto; diseño sustentable; humanismo proyectual; materiales tradicionales; tradiciones indígenas.

\section{Abstract}

The low recognition of identity and aesthetics present in the craft industry and the use of Caña flecha (Gynerium sagittatum) by the Zenú indigenous cultures, highlight the need to inquire about how to understand the dialogue between design, craftsmanship and industrial production processes oriented towards the innovation of methods and products in a context of sustainability. Through a bibliographic review organized in two phases, - the first consisting of the search for scientific articles and books and the second in the analysis thereof -, allowed the classification of information into two categories: I) Design, Handcraft and New trends, and 2) Design and Decolonization. As a result, critical aspects are shown in the relationship between design, crafts and indigenous knowledge. This search discusses the potential that, from design, makes it possible to generate innovation processes. Also, presents contemporary design as a discipline of synthesis that responds to a culturalist or essentialist approach to design, with great empowerment and emancipation capabilities in peripheral contexts. All of this led to question which methodologies allow to approach the understanding of the legacy of cultures and craft processes.
\end{abstract}

Keywords: crafts; subject-centered design; sustainable design; projective humanism; traditional materials; indigenous traditions

\section{Introducción}

Este artículo hace parte de la investigación titulada Técnicas tradicionais indígenas para o desenvolvimento de produtos de Design sustentável com Gynerium sagittatum, desarrollada en el doctorado ${ }^{1}$ en Design del PPGDesign de la Universidad Estatal Paulista (UNESP), Campus de Bauru, Brasil, y en la cual se propone explorar cuestionamientos respecto a: ¿Cómo las técnicas tradicionales de los indígenas zenúes de Colombia pueden contribuir al desarrollo de métodos para el procesamiento de Gynerium Sagittatum empleando conceptos de diseño sustentable?, ¿Cómo dichos procesos pueden contribuir a la generación de materiales para ser aplicados en el diseño de mobiliario empleando conceptos de sustentabilidad? Se tiene como hipótesis que las técnicas tradicionales de los indígenas zenúes son un gran insumo para el desarrollo de nuevos procesos y productos de Gynerium sagittatum empleando conceptos de sustentabilidad, con una ligación a la identidad, el lugar y lo local.

En este artículo se presentan algunas de las indagaciones que forman parte de la búsqueda documental de la investigación, la cual comparte estrategias metodológicas con otros trabajos publicados (Martínez, Barana, Rocha \& Paschoarelli, 2017; Martínez, Paschoarelli \& Da Cruz Landim, 2018; Martínez, Da Cruz Landim

1 Agradecemos al Departamento Administrativo de Ciencia, Tecnología e Innovación (Colciencias) y al Departamento de Sucre, Colombia, por el apoyo con la beca obtenida dentro de la convocatoria para la formación de capital humano de alto nivel para el departamento de Sucre (2014). 
\& Barata, 2018), además de centrar la atención en importantes reflexiones contemporáneas respecto a la relación, cada vez más fuerte, entre diseño y artesanado en el contexto contemporáneo, y en el cual existe un interés creciente por generar productos con un alto componente de innovación para atender las cuestiones sobre identidad, estética y sustentabilidad. Se propone, a partir del panorama identificado, un interrogante sobre las posibilidades de trabajo con los valores implícitos en la artesanía zenú de Colombia y el potencial que, desde el diseño, puede generar procesos de innovación a partir de los conocimientos de las comunidades indígenas.

Una definición muy escueta de lo que se considera artesanado es la que lo supone como un oficio en el cual se desarrollan, principalmente, objetos de uso doméstico, realizados con instrumentos elementales y, en gran parte, a través del uso de las manos.

En muchos contextos, generalmente aquellos de países "periféricos" o "económicamente menos favorecidos" (Bonsiepe, 2011, p. 21), como es el caso de los de Latinoamérica, el artesanado se encuentra ligado fuertemente a una larga tradición que se remonta a los pueblos indígenas, de los cuales ha heredado técnicas, el uso de determinados materiales, informaciones de carácter estético y simbólico y los rasgos distintivos de su visión de mundo y de su identidad.

Históricamente, se tuvo una visión economicista del artesanado, al cual se asumió como algo atrasado, como parte del pasado (Barbosa, 1983, p. 49), carente de valor práctico, más bien exótico, muy poco relacionado con los procesos industriales capitalistas. Hoy en día, esa visión es discutida, por una parte, tomando en cuenta aspectos relacionados con una producción más amable con el ambiente; por otra, debido a la necesidad, cada vez más urgente, de generar procesos innovadores, que puedan diferenciar los productos en un mercado altamente competitivo.

En ese sentido, el artesanado actualmente despierta interés, con la intención de llevar los significados, las tradiciones y la identidad cultural de las comunidades, para hacer parte de procesos de desarrollo de nuevos productos industriales. En este tipo de propuestas, el artesanado cumple un papel desencadenador del saber colectivo; en cierta medida, como una marca de origen que individualiza determinadas líneas de producción industrial (De Vives, 1983, p. 134).

Un ejemplo interesante en ese interés por resignificar la relación entre diseño y artesanado se encuentra en las propuestas que recoge el documento Designers meet artisans. A practical guide, desarrollado por UNESCO (2005), y en el cual se presentan distintas exploraciones que reinterpretan el papel del diseño, como un puente para relacionar los conocimientos de los artesanos y su saber hacer, y el cual se observa cada vez más desconectado de las necesidades y los intereses de los consumidores; así también, con el fin de establecer caminos para llenar el vacío en cuanto a las referencias de un contexto cultural de los productos en un mercado global homogenizado (p. 4).

Otro ejemplo de las teorías de la relación entre el diseño y el artesanado se encuentra en la idea de ejercer el diseño como práctica libertaria (Bonsiepe, 2011), muy cercano a los planteamientos de Manzini (2009), quien se refiere a los conocimientos necesarios para el nuevo papel del diseño en la búsqueda de un desarrollo local y regional. Manzini propone resignificar la visión que se tiene sobre el artesanado, así como promover nuevas maneras de producir, diseñar y enseñar el diseño de forma "no occidentalizante", al igual que generar nuevas herramientas y reflexiones desde el diseño "para estimular y direccionar discusiones estratégicas" (Manzini, 2009, pp. 11-12), un conocimiento que guíe una nueva práctica del diseño.

Arturo Escobar (2016) engloba diferentes conceptos asociados a la idea de una práctica libertaria del diseño, desde una nueva reorientación ontológica, en lo que dicho autor llama diseño para la autonomía. Este se caracteriza, entre otros aspectos, por tener como objetivo la realización de lo comunal; acoger lo ancestral tanto como nuevos futuros posibles; privilegiar intervenciones para el fomento de formas de organización no patriarcal, no liberal, no centrada en el Estado y no capitalistas; considerar la articulación comunidad-actores sociales-tecnologías heterónomas; abierto a concepciones pluriversales para hacer prosperar la vida. El diseño para la autonomía da esperanza a la rebelión en defensa de los principios de la vida (Escobar, 2016, p. 214).

Escobar (2016) recoge de forma interesante lo que se puede considerar un enfoque pertinente para establecer un diálogo entre diseño, artesanado y conocimiento indígena, debido a que articula el respeto por la tradición y el desarrollo de nuevas alternativas en la construcción de futuros posibles, en armonía entre lo humano y lo natural.

Dentro de esos procesos de reinterpretación y sinergia entre el artesanado y la industria, el diseño está llamado a ser una disciplina donde convergen diversos conocimientos, cumpliendo un papel mediador en la asimilación de los significados y los procesos dentro del artesanado, en su adaptación a las metodologías de producción industrial a las que es convocado como recurso para la innovación y la diferenciación de productos contemporáneos.

El interés en el presente trabajo por estudiar las posibilidades de innovación a partir del conocimiento indígena, específicamente hablando de la comunidad indígena zenú de Colombia, se relaciona con la debilidad identificada en la capacidad de investigación y desarrollo tecnológico en 
$\nexists$ Figura 1. Cultivo de caña flecha (Gynerium sagittatum) en Sampués, Sucre, Colombia. Fuente: elaboración propia (2015).

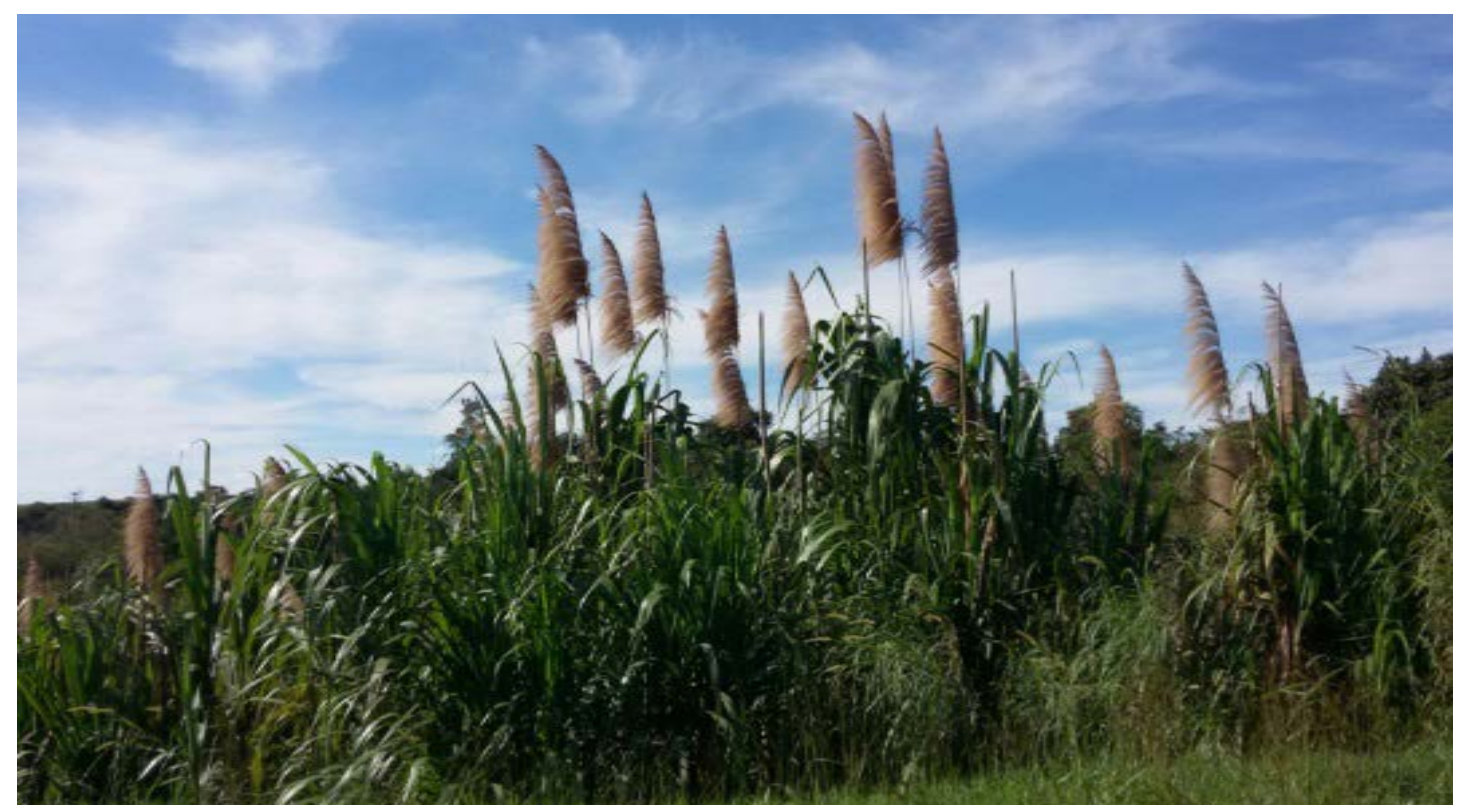

comunidades indígenas en el departamento de Sucre, y en Colombia en general (lo cual puede aplicarse también al ámbito latinoamericano), como herramienta para potencializar el desarrollo local de esos grupos humanos, a fin de aportar de esa forma al desarrollo sustentable de la región y del país.

En la actualidad, en el departamento de Sucre, Colombia, sobreviven grupos indígenas descendientes de la gran familia zenú, que aún mantienen vivas algunas de las tradiciones de su cultura. Están organizados en cabildos indígenas y comandados por un cacique, y se dedican, en su mayoría, a la fabricación de artesanías con fibras de caña flecha (Gynerium sagittatum) (figura 1). Las comunidades indígenas zenúes del departamento de Sucre sobreviven en unas condiciones muy precarias de desarrollo humano, luchando por superar la pobreza extrema y la recuperación de su cultura. En general, padecen problemas de accesibilidad a servicios básicos, así como la carencia de vivienda digna y de buenas y suficientes tierras para cultivar.

Existen algunas iniciativas y estrategias del gobierno de Colombia para atacar brechas estructurales en esas comunidades, como, por ejemplo, la aplicación de un sistema de educación indígena para el pueblo zenú (SEIP) o la inclusión en programas como Familias en Acción (Gobierno de Colombia, 2018). Algunos de dichos intentos para aportar a la superación de las brechas estructurales de las comunidades indígenas, si bien generaron cambios positivos, provienen de políticas con un enfoque asistencialista del desarrollo, las cuales, desde ciertas miradas, pueden limitar el alcance por parte de las comunidades de un desarrollo posible desde sus propios intereses, pues tienen muy poco acceso a la comprensión de los procesos de innovación y desarrollo que desde lo local puedan apalancar sus propias potencialidades.

Tomando en cuenta las necesidades descritas, se pretende generar una reflexión desde el dise- ño, así como su responsabilidad social, en otro sentido del planteado por esta disciplina, la cual se ha enfocado, en gran medida, en el desarrollo de productos para el consumo de moda; en vez de este último, se propende por el sentido que plantea Bonsiepe (2011) cuando habla del diseño como la "solución inteligente de problemas" y la capacidad de este para construir un mundo diferente, un "humanismo proyectual", entendido como "El ejercicio de las capacidades proyectuales para interpretar las necesidades de grupos sociales y elaborar propuestas viables, emancipatorias, en forma de artefactos instrumentales y artefactos semióticos" (Bonsiepe, 2011, p. 21).

En el departamento de Sucre, a pesar de las debilidades y las problemáticas identificadas, existen unas potencialidades que pueden aprovecharse para generar desarrollos que puedan impulsar el mercado, ya sea interno o externo, según las condiciones que se generen. Entre otras potencialidades, se puede identificar una tradición cultural ancestral que aún sobrevive en las comunidades indígenas, en torno a la caña flecha; sobre todo en cuanto a la producción de artesanías desde el diseño, son muy pocos los estudios en pro de encontrar vías alternativas para el desarrollo y la innovación desde el uso de esta tradición, lo cual es un escenario de trabajo e investigación muy prometedor para generar procesos y productos innovadores.

\section{Metodología}

Este artículo hace parte de la búsqueda de referentes en el proceso de justificar y dar soporte a la propuesta de investigación que integra temáticas inherentes a la relación entre diseño, artesanado y conocimiento indígena. La metodología de tipo cualitativo desarrollada para esta revisión bibliográfica, y que es la fase preliminar de una investigación más amplia, tuvo como propósito identificar los abordajes investigativos recientes sobre la relación entre el diseño y el artesanado. 
La revisión se llevó a cabo en dos fases: una primera fase donde se revisaron artículos científicos y libros, producto de investigación en las bases de datos ScienceDirect, Scopus, Proquest, JSTOR-Art \& Sciences II, EBSCOhost. Se establecieron como palabras clave en la búsqueda: "design" y "handcraft," y se incluyeron solo los documentos que cumplieran con los filtros que se establecieron: journals, books, Open Access articles.

Los criterios de selección de los documentos identificados fueron: 1) design-enfoque colaborativo, 2) design-intervenciones comunidades indígenas, y 3) Handcraft-nuevas tendencias de trabajo. Se adoptaron esos criterios debido a que se relacionan directamente con los intereses de la propuesta en construcción.

La segunda fase consistió en el análisis de la información recopilada en fichas de lectura de los artículos seleccionados como más relevantes por los temas abordados. Para el estudio de los documentos se tuvieron en cuenta como elementos de análisis: el concepto sobre la relación diseño-artesanado, los materiales y los métodos utilizados, y el papel de las comunidades involucradas en los procesos investigativos. La búsqueda se complementó con la ampliación, específicamente, de las temáticas: "design-decolonization", "design-empowerment" y "design-Gynerium sagittatum", a través de la revisión de referencias bibliográficas extraídas de los artículos, a fin de conseguir un panorama teórico más amplio.

Finalmente, se clasificó la información encontrada en dos categorías: 1) Design-Handcraft, nuevas tendencias, y 2) Design-Decolonization, las cuales permitieron acercarse a una comprensión general del panorama identificado.

\section{Resultados}

\section{Diseño y artesanado: los abordajes contemporáneos}

En los últimos años, el artesanado despertó el interés de los procesos de producción industrial, debido a las condiciones prometedoras que este ofrece para la innovación. Se explora, con esa nueva perspectiva, su potencial comunicacional haciendo posible un diálogo en doble vía entre artesanos y diseñadores (Yair, Press \& Tomes, 2001, p. 384).

En el camino de construcción de ese nuevo diálogo entre diseño y artesanado, se habla de la generación de un nuevo conocimiento que sale desde el grupo de diseño hacia el equipo de producción. Yair et al. (2001, p. 393) hablan sobre cómo ese conocimiento puede ser transferido generando competencias estratégicas y productos competitivos, debido a su gran diferenciación en el mercado y su difícil emulación, lo cual, en muchos casos combinado con desarrollos tecnológicos, les permite a las empresas satisfacer las necesidades de sus clientes al incorporar un alto valor en los productos, y así ser más competitivos en un mercado globalizado (Linzalone, 2008, p. 38).

Se realizan cada vez más ejercicios en los cuales se exploran las posibilidades de ese diálogo entre diseño y artesanado, como, por ejemplo, el estudio desarrollado por Alexandre, Gómez y Valente, donde se exploran mecanismos para relacionar a diseñadores y artesanos con el fin de mejorar y revitalizar el trabajo de los artesanos implementando en el proceso de producción, los recursos y los conceptos de la industria enfocada en nuevas tecnologías (computer aided technologies [CAX]), con el fin de explorar el establecimiento de bases sostenibles entre diseñadores y artesanos en el contexto de un trabajo colaborativo (2015, p. 1089).

En un trabajo colaborativo desarrollado por Tung (2012), se propone el diseño como catalizador del conocimiento artesanal hacia otras industrias creando sinergias para estimular el desarrollo de lo local y tomando en cuenta tanto la sostenibilidad como aspectos comerciales y de mercado:

\footnotetext{
The fusion of craft and design manifests a design praxis and model which in turn presents a promising strategy for product development. Hence, engaging designers to collaborate with craftspeople is not only helpful for the regeneration of traditional crafts, but it also allows designers to obtain new design experiences, which could broaden their design horizons. (p. 71-74) ${ }^{2}$
}

En el sentido que plantea Tung, es posible una integración entre diseño y artesanado como una estrategia innovadora y promisoria para el desarrollo de productos, a la vez que como una posibilidad interesante de expandir los horizontes y los límites que preexisten en el diseño.

En el proceso de cocreación que describe Tung, se proponen dos maneras de aproximación entre el diseño y artesanado. Una es la introducción de métodos adecuados de manufactura en los procesos de producción, y otra es explorar nuevas técnicas alternativas a través de la intuición y la experimentación (Tung, 2012, p. 77).

Este creciente interés por el trabajo colaborativo entre diseño y artesanado puede generar posiciones encontradas; sobre todo, en relación con el debate sobre la identidad en la contemporaneidad. Por una parte, al defenderse la ruptura con el pasado y la generación de nuevas identidades en la búsqueda de la innovación (Yagou, 2007; Kalyan, 2011); por otra, cuando se

\footnotetext{
2 La fusión de la artesanía y el diseño manifiesta una praxis y un modelo de diseño que a su vez presenta una estrategia prometedora para el desarrollo de productos. Por lo tanto, involucrar a los diseñadores para que colaboren con los artesanos no solo es útil para la regeneración de las artesanías tradicionales, sino que también les permite a los diseñadores obtener nuevas experiencias de diseño, lo que podría ampliar sus horizontes de diseño.
} 

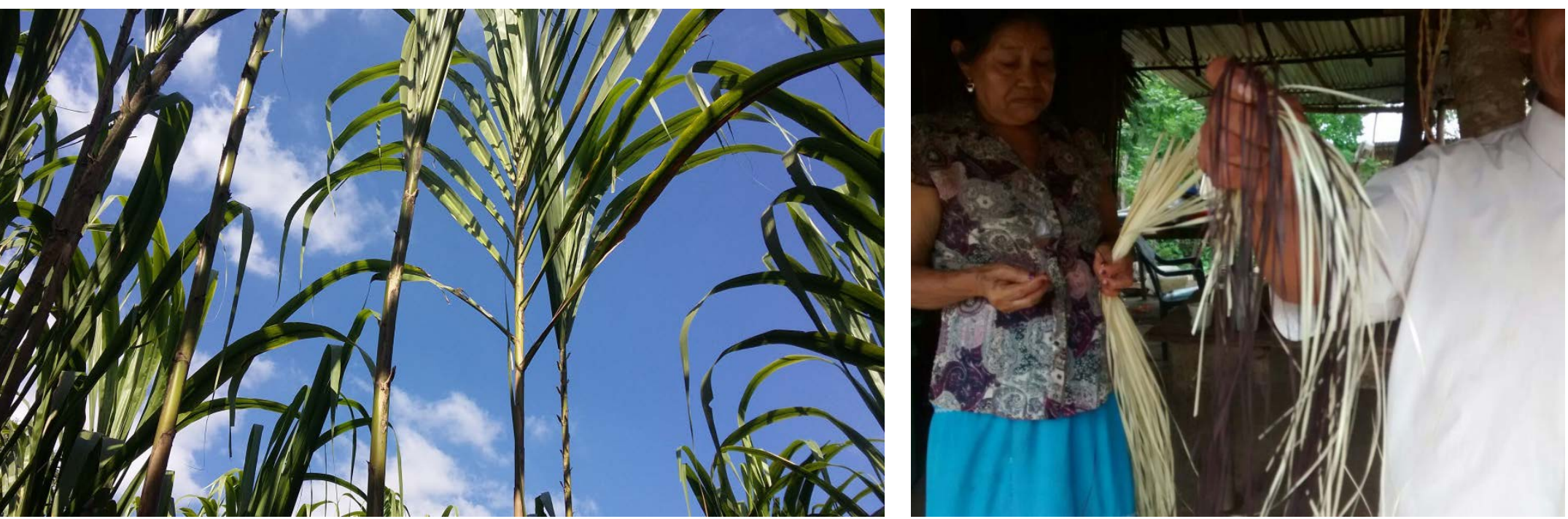

(A) Figura 2. Caña flecha (Gynerium sagittatum) en Sampués, Sucre, Colombia. Fuente: elaboración propia. (2016)

(A) Figura 3. Artesanos indígenas en Sampués, Sucre, Colombia.

Fuente: elaboración propia (2017). procura generar contactos con el pasado, en busca de apropiación e identidad local y regional, como una reafirmación y una forma de diferenciación en el mercado global (De Castro, 2007; Ota, 2012; Cardoso, 2012).

Son interesantes las investigaciones enfocadas en el sentido de la búsqueda de identidad para el desarrollo de productos con la estrategia diseño y artesanado; sobre todo, las desarrolladas en torno a la resignificación y la reinterpretación de las posibilidades de los materiales tradicionalmente utilizados en el artesanado. Tal es el caso de los desarrollos tecnológicos en relación con los nuevos usos del caucho, el coco, el bambú y otros residuos agrícolas como sustitutos de la madera para el desarrollo de nuevos productos (Durst, Killmann \& Brown, 2004, p. 47), (Pereira \& Beraldo, 2008, p. 40), (Jarusombuti, Hiziroglu, Bauchongkol \& Fueangvivat, 2009; Esteve-Sendra, Moreno-Cuesta, Portalés-Mañanós \& Magal-Royo, 2012, p. 777).

En los mencionados estudios se habla de una iniciativa interesante para abordar el reto de una producción más sostenible, no solo con la perspectiva ambiental, sino también vista desde un compromiso para contribuir a generar un cambio social de fuertes repercusiones en la estructura económica. A su vez, proponen un camino prometedor a la hora de abordar, desde el diseño, problemas estructurales en el mundo contemporáneo.

\section{Una propuesta desde el diseño contemporáneo como aproximación al conocimiento indígena en el artesanado zenú}

A escala global se puede observar un incremento en la necesidad de responder de mejor forma a los problemas ambientales, así como un creciente interés en lograr conexiones que ayuden a proteger y conservar conocimientos ancestrales de los indígenas en un contexto cada vez más globalizado (Kotze \& Traynor, 2011, p. 272).
Pacheco, Barrero y Gómez Vásquez (2013) describen trabajos desarrollados en Colombia utilizando la estrategia de articulación entre diseño y artesanado, cuyo enfoque radica en usar estrategias ecotecnológicas en el proceso de producción, para lograr un manejo sostenible de los recursos naturales y mejorar así el desbalance tecnológico en relación con la participación sostenible de la producción del artesanado en los mercados globales (Pacheco et al., 2013, p. 120).

En el sentido del enfoque tecnológico abordado en los procesos de articulación entre diseño y artesanado, se plantea aquí una propuesta de investigación para abordar cuestiones relativas al rescate de la identidad de la comunidad de artesanos descendientes de la etnia zenú, comunidad indígena de la región norte de Colombia, debido a la importancia del legado cultural de esta comunidad, evidenciado en los productos artesanales elaborados a partir de la utilización de Gynerium sagittatum (figura 2), los cuales son patrimonio y símbolo nacional que identifica la producción artesanal de Colombia en el mundo.

Son diversos los trabajos que se han desarrollado en torno a las posibilidades de la caña flecha, o caña brava (Gynerium sagittatum), como material sustituto de la madera para el desarrollo de nuevos productos forestales. Tal es el caso de los desarrollos en el tema por parte de Contreras y Owen (1997), Contreras, Owen, Cloquell y Contreras (2004), Contreras, Cloquell y Owen (2008; 2010), Contreras y Owen (2011) y Miranda (2005), sobre laminados de caña brava y la producción de tableros de gramíneas y otros residuos vegetales, incluyendo caña brava, así como la fabricación de elementos estructurales tipo Parallam a base de laminados de caña brava. Todo ello manifiesta el interés por parte de la comunidad científica en este material y su potencial para el desarrollo de productos forestales.

Se propone, a partir de las problemáticas identificadas y los referentes encontrados, la investigación denominada Técnicas tradicionais indígenas para o desenvolvimento de produtos de Design sustentável com Gynerium sagittatum. 
Se busca con esa investigación generar nuevas posibilidades de desarrollo de productos a partir de la reinterpretación de los conocimientos de comunidades indígenas (figura 3) (Martínez, Da Cruz \& Barata, 2018), la construcción de diálogos significativos entre diseño y artesanado, enfocados en la creación de sinergias y el empoderamiento de las comunidades periféricas desde un nuevo posicionamiento tecnológico, y cuestionando desde el diseño los procesos de colonización y poscolonialismo que desencadenaron el sistema de mundo vigente, y que ha determinado la historia de América Latina (Quijano, 2000, p. 246).

En esta investigación, el diseño desde las reflexiones contemporáneas sobre tecnología, identidad y globalidad ataca problemas estructurales en la región Caribe colombiana, como las pocas capacidades de investigación y de desarrollo tecnológico en comunidades indígenas en el departamento de Sucre, como un obstáculo que impide potencializar el desarrollo local de esos grupos humanos. Se pretende también impactar en la cadena productiva de la madera en Colombia, la cual requiere con urgencia investigar e innovar sobre la transformación y la generación de valor agregado para aprovechar las oportunidades que brinda el mercado nacional y el internacional para este sector, para aportar de esa forma al desarrollo sustentable de la región y del país.

\section{Discusión}

Desde el panorama identificado surge una serie de interrogantes que abren el camino a una discusión interesante, por una parte, en cuanto a la relación entre diseño y artesanado, y, por otra, en torno a la relación entre diseño, identidad y conocimiento indígena. Una primera reflexión sería en el sentido de analizar qué motiva el creciente interés de la industria y el diseño contemporáneo para relacionarse con técnicas y conocimientos ancestrales y populares.

Muchos de los ejemplos identificados enfatizan la importancia de la participación en procesos colaborativos de diseño; sobre todo, por su capacidad de empoderar a los participantes (Saskia, 2015, p. 239). En tal sentido, algunas de esas iniciativas de empoderamiento a comunidades de artesanos vienen acompañadas por incentivos gubernamentales hacia la innovación como una variable importante para ser tenida en cuenta en los procesos de producción, ya que logra una mayor competitividad frente a las necesidades cambiantes de un mercado globalizado (Jiménez Castañeda, Domínguez Hernández, \& Martínez Castro, 2009, p. 8), lo cual también permite estrategias de mercado justo, como alternativas éticas a las prácticas de mercado neoliberales (Alvarado, 2009, p. 301).

Por otra parte, Campbell (2014) busca entender ese interés desde otra óptica, al plantear la necesidad de la industria contemporánea de personalizar y dar valor agregado a sus nuevos productos, lo cual es difícil de conseguir simplemente con estrategias innovadoras, pues la industrialización, pese a tener múltiples posibilidades de diferenciación, muy difícilmente contiene una carga de memorias y significados que son inherentes al artesanado (Campbell, 2014, p 56).

Sudjic (2010) pone especial énfasis en esa capacidad comunicativa de los objetos y explica el interés del diseño contemporáneo en trabajar con el carácter emocional de los objetos:

\section{Vivemos numa época em que nossa relação com aquilo que possuímos está passando por uma transformação radical. Desde que surgiu como profissão independente o design é usado para manipular o desejo. (p. 21) ${ }^{3}$}

Esto que plantea Sudjic, posiblemente, da indicios sobre algunos intereses que pueden influir en las motivaciones de la industria del diseño en acercarse al artesanado en busca de sus potencialidades.

Otras reflexiones sobre el contexto identificado, en el sentido de entender el diálogo que se construye entre diseño y artesanado en su asimilación a los procesos de producción industrial, serían: ¿cómo se aprehende ese proceso en cada uno de los actores? ¿Qué situaciones se generan en el proceso?

Taffe (2015) cuestiona los procesos colaborativos en el diseño planteando diferentes paradojas en el contexto de procesos de cocreación en los cuales el diseñador trabaja colaborativamente con usuarios finales del producto que se va a desarrollar. Propone al respecto tres paradojas: 1) la paradoja del usuario rechazando diseños para sí mismo, 2) la paradoja del usuario actuando como diseñador y, finalmente, 3) la paradoja del usuario diseñando para usuarios ideales en colaboración con el diseñador (Taffe, 2015, pp. 47-52).

Esa reflexión de Taffe (2015), aunque en un contexto distinto del contexto del trabajo colaborativo entre diseñadores y artesanos, refleja aspectos de interés que pueden presentarse en el proceso de cocreación entre diseño y artesanado. Algunos de dichos aspectos son interesantes en el sentido de empoderar al artesano, pero en otros se necesita la cautela, ya que es preciso siempre tener como foco problemas reales a los cuales se busca una óptima solución.

Campbell (2014, p. 61) habla de diseño emancipador refiriéndose a las posibilidades del diseño de empoderar comunidades; en este caso, comunidades indígenas, artesanos, comunidades periféricas que, a través de procesos de cocreación, son estimulados en su proceso autónomo de desarrollo. Tromp y Hekkert (2016) plantean

3 Vivimos en una época en que nuestra relación con aquello que poseemos está experimentando una transformación radical. Desde su surgimiento como profesión independiente, el diseño se ha utilizado para manipular el deseo. 
algo similar en relación con las implicaciones sociales del diseño, desde las cuales el diseñador es impulsado a diseñar productos servicios que faciliten el cambio actitudinal en el contexto ( $p$. 27). En el mismo sentido, Wiltschnig y Christensen (2013) consideran los procesos de cocreación algo necesario para estimular la práctica cotidiana del diseño.

Otros cuestionamientos interesantes para ser discutidos serían: ¿cuáles metodologías son apropiadas para generar ese diálogo entre diseño y artesanado? ¿Cuáles metodologías permiten acercarse a la comprensión del legado de las culturas aborígenes inherente al artesanado? ¿Cuáles enfoques permiten desde el diseño abordar esos procesos metodológicos?

El mundo contemporáneo, como un contexto complejo, hace posibles diferentes miradas y enfoques con los cuales generar diálogos con el pasado y la identidad cultural de los pueblos. Un ejemplo se puede encontrar en el trabajo de Busato (2013), en el que se explora el papel del diseño como disciplina de síntesis, capaz de apropiar conocimientos disímiles para luego utilizarlos como herramientas aplicadas al proyecto (p. 10).

Busato plantea el desarrollo de una metodología basada en la observación, que toma elementos de los desarrollos en la semiótica, la neurociencia y la metaciencia, con una perspectiva integradora de la complejidad en el diseño, lo que le permite generar conclusiones en distintos espacios transdisciplinares, y así crear relaciones emergentes desde el diseño.

Una metodología integradora como la que desarrolla Busato (2013) es de gran interés para abordajes contemporáneos, como el que se plantea en este trabajo, en el sentido de generar diálogos que permitan entender problemáticas complejas como las que hacen parte de una búsqueda que relaciona diseño, artesanado, conocimientos y técnicas tradicionales de comunidades indígenas de Colombia.

Un trabajo de investigación como el que se plantea está en sintonía con un enfoque culturalista o esencialista del diseño, del que habla Bonsiepe (2011, p. 63), y que, al contrario de una visión romántica de pasado, propone una posición que busca la autoafirmación de estrategias de desarrollo en los términos y dentro de las posibilidades de las periferias. Se abre a posibilidades tecnológicas que generen una transformación en contextos históricamente subvalorados y discriminados. En palabras de Papanek, "diseñar por las necesidades de la humanidad", "diseño para el hombre y no para el dinero" (1985, p. 69).

\section{Conclusiones}

Es posible plantear algunas conclusiones preliminares a partir del panorama identificado, en el sentido de enfocar y posicionar la propuesta de investigación que se proyecta en relación con los interrogantes contemporáneos sobre diseño y artesanado.

Es significativo el reciente interés por generar diálogos entre el diseño y el artesanado, lo cual está ligado a diferentes intereses y necesidades. El diseño debe aprovechar esa situación especial que ofrece el contexto contemporáneo y generar reflexiones que lo posicionen nuevamente como una disciplina con fuertes capacidades para generar discusiones y cambios estructurales en la sociedad. El diseño para el ser humano debe ser la premisa ineludible.

En la búsqueda de soluciones inteligentes para los problemas humanos, el mundo de hoy, como un contexto cambiante, diverso, heterogéneo y complejo, exige a los diseñadores repensar sus estrategias de trabajo, sus enfoques y sus potencialidades. Es cada vez más necesario desmontar preconceptos y traspasar barreras disciplinarias, con el fin de generar relaciones y diálogos significativos que ayuden a transformar de manera positiva la realidad.

El proyecto de investigación que se propone, en cuanto a la relación entre diseño, artesanado y conocimiento de comunidades indígenas, es una propuesta sobre un tema relevante y de gran interés en la contemporaneidad. Se posiciona en un campo del diseño, en el cual es pensado como una disciplina capaz de establecer sinergias que ayuden a resolver problemáticas de fondo; diseño como instrumento de emancipación. 


\section{Referencias}

Alexandre, C. B., Gómez, E. A., \& Valente, A. C. (2015). Interdisciplinary relationship between Designer and Craftsman based on Integrated Craft Manufacturing Systems. Procedia Engineering, 132, 1089-1095.

https://doi.org/10.1016/j.proeng.2015.12.600

Alvarado, J. (2009). Fair Trade in Mexico and Abroad: An Alternative to the Walmartopia?. Journal of Business Ethics, 88, 301-317. https://doi.org/10.1007/s10551-009-0287-6

Barbosa, M. (1983). Artesanato, tradição e mudança social - um estudo a partir da "arte do oro" de Juazeiro do norte. En Ribeiro B.G, et al., O artesão tradicional e seu papel na sociedade contemporânea (pp. 49-100). Rio de Janeiro: Funarte, Instituto Nacional do Folclore. Recuperado de:: https://searchworks.stanford.edu/view/1522799.

Bonsiepe, G. (2011). Design, cultura e sociedade. São Paulo: Blucher.

Busato, S. (2013). Visão e percepção investigação dos modelos cognitivos - uma abordagem neurobiológica em design e emoção. PhD dissertation, Universidade Estadual Paulista. Faculdade de Arquitetura, Artes e Comunicação, Bauru. Recuperado de: http://repositorio.unesp.br/handle/11449/89356

Campbell, W. (2014, september). Designs: An Archaeology of "Craft" as God Term. Computers and Composition 33, 50-67. https://doi.org/10.1016/j.compcom.2014.07.002

Cardoso, R. (2012). Design para um mundo complexo. São Paulo, Brasil: Cosac Naify.

Contreras, W., Cloquell, V., \& Owen, M. (2008, enero-junio). Determinación de los niveles de sostenibilidad del proceso de fabricación de tableros de caña brava (Gynerium Sagittatum), a partir del método acv-Coclowen. Revista Forestal Venezolana, XLII, 52(1). 47-59. Recuperado de::

http://www.saber.ula.ve/handle/123456789/29085

Contreras, W., Cloquell, V., \& Owen de C. M. (2010). Las técnicas de decisión multicriterio en la selección de componentes estructurales, a partir de la tecnología de la madera, para construcción de viviendas sociales en Venezuela. Revista Madera y Bosques, 16 (3), 7-22.

https://doi.org/10.21829/myb.2010.1631163

Contreras, W., Owen de C. M., Cloquell, V., \& Contreras, Y. (2004). Generación de nuevos productos forestales para sistemas estructurales a partir de gramíneas y residuos de plantación de pino caribe (Pinus caribaea var. hondurensis). En Ponencia DPI-11. VII Congreso AEIPRO, 6 (8), octubre de 2004. Recuperado de::

http://mww.aeipro.com/files/congresos/2004bilbao/ ciip04_0066_0075.1203.pdf

Contreras, W., \& Owen de C. M. (1997). Elaboración de un elemento estructural laminado, tipo parallam, con tiras de caña brava gynerium sagittatum y adhesivo fenol-formaldehido. Revista Forestal Venezolana 41(1), 29-36. Recuperado de:

http://www.saber.ula.ve/handle/123456789/4909

Contreras, W., \& Owen, H. (2011). El Eco diseño y su rol protagónico en la construcción de una sociedad venezolana sostenible. Seminario de construcción y desarrollo sostenible. Caracas, Venezuela. Recuperado de:: http://docplayer.es/9397726-El-ecodisenoy-su-rol-protagonico-en-la-construccion-deuna-sociedad-venezolana-sostenible.html

De Castro, J. (2007, abril). Design com identidade: por meio de estudos sócio-culturais e dos signos. MsC dissertation. Programa de Pós-Graduação em Desenho Industrial da Universidade Estadual Paulista, Faculdade de Arquitetura, Artes e Comunicação, Campus de Bauru. Recuperado de:

http://www.faac.unesp.br/Home/Pos-Graduacao/ Design/Dissertacoes/jacquelinedecastro.pdf
De Vives, V. (1983). A beleza do cotidiano. En Ribeiro et al., $O$ artesão tradicional e seu papel na sociedade contemporânea (pp. 132-163). Rio de Janeiro: Funarte, Instituto Nacional do Folclore. Recuperado de: https://searchworks.stanford.edu/view/1522799

Durst, P. B., Killmann, W., \& Brown, C. (2004). Asia's new woods. Journal of forestry, 102(4). 46-53. Recuperado de:: https://academic.oup.com/jof/article/102/4/46/4613195

Escobar, A. (2016). Autonomía y diseño: La realización de lo comunal. Popayán: Universidad del Cauca.

Esteve-Sendra, C., Moreno-Cuesta, R., Portalés-Mañanós, A., \& Magal- Royo, T. (2012). Bamboo, from traditional crafts to contemporary design and architecture. Procedia - Social and Behavioral Sciences, 51 (2012), 777-781.

Gobierno de Colombia (2018). Programa Familias en acción. Recuperado de: http://www.prosperidadsocial.gov.co/que/ fam/famacc/Paginas/default.aspx

Jarusombuti, S., Hiziroglu, S., Bauchongkol, P., \& Fueangvivat, V. (2009). Properties of Sandwich-Type Panels Made from Bamboo and Rice Straw. Forest Products Journal, 59(10), 52-57.

Jiménez Castañeda, J. C., Domínguez Hernández, M. L., \& Martínez Castro, C. J. (2009). Estrategias y competitividad de los negocios de artesanía en México. Pensamiento \& Gestión, (26), 165-190. Recuperado de:: http://www.scielo.org.co/scielo.php?script=sci arttext\&pid =S1657-62762009000100008\&lng $=$ en\&tlng $=\mathrm{es}$

Kalyan, R. (2011). Fragmentation by Design: Architecture, Finance, and Identity. Grey Room, 44, 26-53. https://doi.org/10.1162/GREY_a_00041

Kotze, D. \& Traynor, C. (2011). Wetland Plant Species Used for Craft Production in Kwazulu-Natal, South Africa: Ethnobotanical Knowledge and Environmental Sustainability. Economic Botany, 65(3), 271-282. https://doi.org/10.1007/s12231-011-9166-z

Linzalone, R. (2008). Leveraging knowledge assets to improve new product development performances. Measuring business excellence, 12(2), 38-50. https://doi.org/10.1108/13683040810881180

Manzini, E. (2009). New design knowledge. Design Studies. 30(1), 4-12 https://doi.org/10.1016/j.destud.2008.10.001

Martínez, P., Paschoarelli, L., \& Da Cruz Landim, P. (2018). Aproximación acerca de los interrogantes sobre lugar e identidad en el diseño contemporáneo. Designia, 5(2), 67-79. https://doi.org/10.24267/22564004.275

Martínez, P., Da Cruz L. P., \& Barata, T. (2018, abril). Procesos artesanales para la producción sostenible de tableros de caña flecha (Gynerium sagittatum). Mix sustentável, 4(2) 59-70.

Doi:https://doi.org/10.29183/2447-3073. MIX2018.v4.n2.59-70

Martínez-Osorio, P., Barana, M., Rocha-Carneiro, R., \& Paschoarelli, L. (2017). Innovación, design y sostenibilidad social: nuevas tendencias para el desarrollo local en la ciudad contemporánea. Revista de Arquitectura (Bogotá), 19(2), 68-77. http://dx.doi.org/10.14718/RevArq.2017.19.2.1406

Miranda, W. C. (2005, junio). El diseño ambientalmente integrado en la propuesta venezolana de tableros de partículas de caña brava y adhesivo fenol-Formaldehído (R10\%/R 13\%). En: Congresos Forestales. Recuperado de:: http://secforestales.org/publicaciones/index. php/congresos/article/viewFile/7556/7479
Ota, M. (2012). Vanguarda sempre. En: De Faria E Silva, R. (ed.), Um olhar sobre o design brasileiro. (Edición revisada) (p. 22-27). São Paulo: SENAI-SP.

Pacheco, J., Barrero, G., \& Gómez Vásquez, G. (2013). An Eco-Technological Approach to Handcraft Production. Two Cases in the Colombian Caribbean Region. Cuadernos de Desarrollo Rural, 10(70), 115-129. Recuperado de: http://www.scielo.org.co/scielo. php?script $=$ sci arttext \&pid $=$ S012214502013000100006\&lng $=$ en\&tlng $=$ en

Papanek, V. (1985). Design for the real world. Human ecology and social change. London: Thames \& Hudson Ltd.

Pereira, M. \& Beraldo, A. (2008). Bambu de corpo e alma. Bauru, SP: Canal6 editora.

Quijano, A. (2000). Colonialidad del poder, eurocentrismo y América Latina. En: Lander, Edgardo (comp.). La colonialidad del saber: eurocentrismo y ciencias sociales. Perspectivas Latinoamericanas. Buenos Aires: CLAC$\mathrm{SO}$, Consejo Latinoamericano de Ciencias Sociales. Recuperado de::

http://bibliotecavirtual.clacso.org.ar/clacso/sursur/20100708034410/lander.pdf

Saskia, V. M. (2015). Co-ideation of disaster preparedness strategies through a participatory design approach: Challenges and opportunities experienced at Turrialba volcano, Costa Rica. Design Studies, 40 (C), 218-245. https://doi.org/10.1016/j.destud.2015.06.002

Sudjic, D. (2010). A linguagem das coisas. Rio de Janeiro: Intrínseca 224

Taffe, S. (2015). The hybrid designer/end-user: Revealing paradoxes in co-design. Design Studies, 40(C), 39-59.

https://doi.org/10.1016/j.destud.2015.06.003

Tromp, N. \& Hekkert, P. (2016). Assessing methods for effect-driven design: Evaluation of a social design method. Design Studies, 43(C), 24-47.

Doi:https://doi.org/10.1016/j.destud.2015.12.002

Tung, F. W. (2012). Weaving with Rush: Exploring Craft-Design Collaborations in Revitalizing a Local Craft. International Journal of Design, 6(3), 71-84. Recuperado de::

www.ijdesign.org/index.php/IJDesign/article/ viewFile/1077/528

UNESCO. (2005). Designers meet artisans. A practical guide. New Delhi, India: Craft Revival Trust, Artesanías de Colombia S.A., UNESCO. Recuperado de::

h t t p : / / u n e s d o c. un e s c o.org/ images/0014/001471/147132EO.pdf

Wiltschnig, S., \& Christensen, B. (2013, September). Collaborative problem solution Coevolution in creative design. Design Studies, 34(5), 515-542.

https://doi.org/10.1016/j.destud.2013.01.002

Yagou, A. (2007). Metamorphoses of Formalism: National Identity as a Recurrent Theme of Design in Greece. Journal of Design History, 20(2), 14-159. Doi: https://doi.org/10.1093/jdh/epm006

Yair, K., Press, M., \& Tomes, A. (2001, July). Crafting competitive advantage: crafts knowledge as a strategic resource. Design Studies, 22(4), 377-394

https://doi.org/10.1016/S0142-694X(00)00043-0 


\section{Vol.}
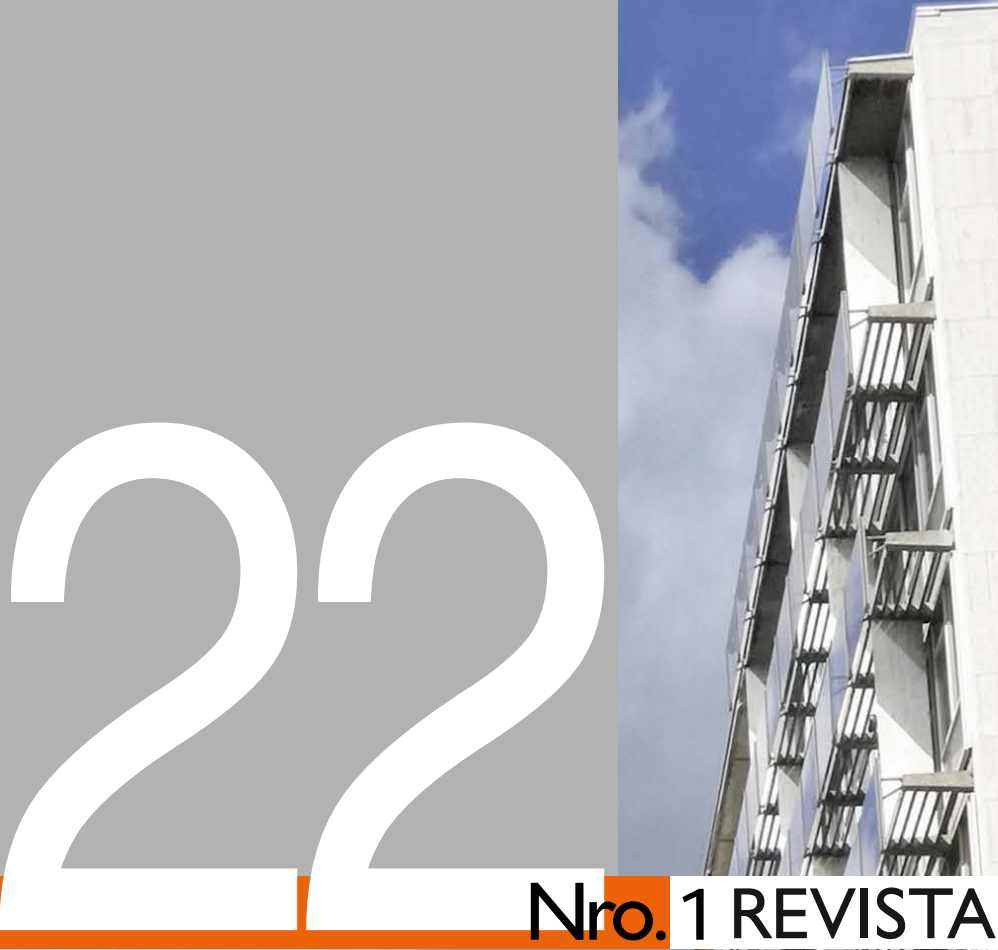

ISSN: 1657-0308 (Impresa)

Nro. 1 REVISTA DE ARQUITECTURA

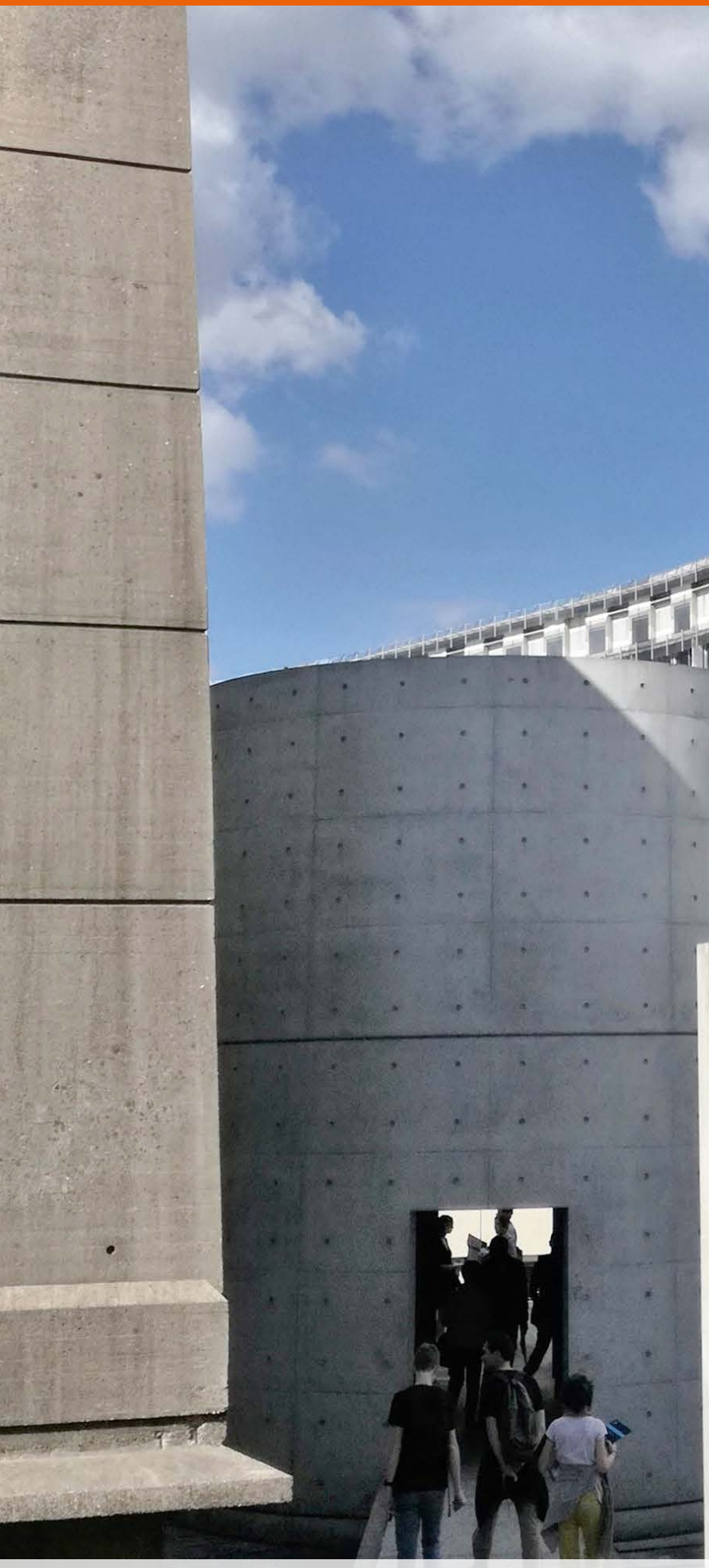

$\overline{9}$
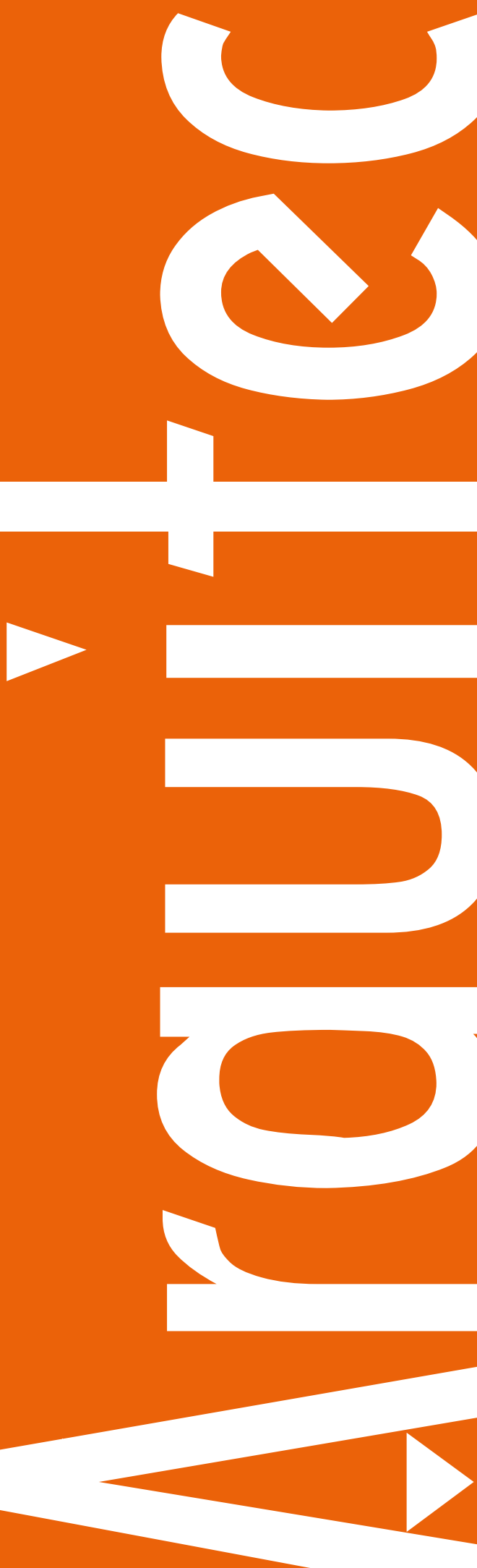


\section{Enfoque y alcance}

La Revista de Arquitectura (Bogotá) ( (ISSN 1657-0308 Impresa y E-ISSN 2357-626X en línea) es una publicación científica seriada de acceso abierto, arbitrada mediante revisión por pares (doble ciego) e indexada, en donde se publican resultados de investigación originales e inéditos.

Está dirigida a la comunidad académica y profesional de las áreas afines a la disciplina. Es editada por la Facultad de Diseño y el Centro de Investigaciones (CIFAR) de la Universidad Católica de Colombia en Bogotá (Colombia).

La principal área científica a la que se adscribe la Revisto de Arquitectura (Bogotá) según la OCDE es:

Gran área: 6. Humanidades

Área: 6.D. Arte

Disciplina: 6D07. Arquitectura y Urbanismo

También se publican artículos de las disciplinas como 2A02, Ingeniería arquitectónica; 5C03, Estudios urbanos (planificación y desarrollo); 6D07, Diseño.

Los objetivos de la Revista de Arquitectura (Bogotá) son:

- Promover la divulgación y difusión del conocimiento generado a nivel local, nacional e internacional

- Conformar un espacio para la construcción de comunidades académicas y la discusión en torno a las secciones definidas.

- Fomentar la diversidad institucional y geográfica de los autores que participan en la publicación.

- Potenciar la discusión de experiencias e intercambios científicos entre investigadores y profesionales.

- Contribuir a la visión integral de la arquitectura, por medio de la concurrencia y articulación de las secciones mediante la publicación de artículos de calidad.

- Publicar artículos originales e inéditos que han pasado por revisión de pares, para asegurar que se cumplen las normas éticas, de calidad, validez científica, editorial e investigativa.

- Fomentar la divulgación de las investigaciones y actividades desarrolladas en la Universidad Católica de Colombia.
Palabras clave de la Revista de Arquitectura (Bogotá): arquitectura, diseño, educación arquitectónica, proyecto y construcción, urbanismo.

Idiomas de publicación: español, inglés, portugués y francés. Título abreviado: Rev. Arquit.

\section{Titulo corto: RevArq}

\section{Políticas de sección}

La revista se estructura en tres secciones correspondientes a las líneas de investigación activas y aprobadas por la institución, y dos complementarias, que presentan dinámicas propias de la Facultad de Diseño y las publicaciones relacionadas con la disciplina.

Cultura y espacio urbano. En esta sección se publican los artículos que se refieren a fenómenos sociales en relación con el espacio urbano, atendiendo aspectos de la historia, el patrimonio cultural y físico, y la estructura formal de las ciudades y el territorio.

Proyecto arquitectónico y urbano. En esta sección se presentan artículos sobre el concepto de proyecto, entendido como elemento que define y orienta las condiciones proyectuales que devienen en los hechos arquitectónicos o urbanos, y la forma como estos se convierten en un proceso de investigación y nuevo de conocimiento. También se presentan proyectos que sean resultados de investigación, los cuales se validan por medio de la ejecución y transformación en obra construida del proceso investigativo. También se contempla la publicación de investigaciones relacionadas con la pedagogía y didáctica de la arquitectura, el urbanismo y el diseño.

Tecnología, medioambiente y sostenibilidad. En esta sección se presentan artículos acerca de sistemas estructurales, materiales y procesos constructivos, medioambiente y gestión, relacionados con los entornos social-cultural, ecológico y económico.

Desde la Facultad. En esta sección se publican artículos generados en la Facultad de Diseño, relacionados con las actividades de docencia, extensión, formación en investigación o internacionalización, las cuales son reflejo de la dinámica y de las actividades realizadas por docentes, estudiantes y egresados; esta sección no puede superar el $20 \%$ del contenido.

Textos. En esta sección se publican reseñas, traducciones y memorias de eventos relacionados con las publicaciones en Arquitectura y Urbanismo.
A Frecuencia de publicación

Desde 1999 y hasta el 2015, la Revista de Arquitectura (Bogotá) publicó un volumen al año, a partir del 2016 se publicarán dos números por año en periodo anticipado, enero-junio y julio-diciembre, pero también maneja la publicación anticipada en línea de los artículos aceptados (versión Post-print del autor).

La Revista de Arquitectura (Bogotá) se divulga mediante versiones digitales (PDF, HTML, EPUB, XML) e impresascon un tiraje de 700 ejemplares, los tiempos de producción de estas versiones dependerán de los cronogramas establecidos por la editorial.

Los tiempos de recepción-revisión-aceptación pueden tardar entre seis y doce meses dependiendo del flujo editorial de cada sección y del proceso de revisión y edición adelantado.

Con el usuario y contraseña asignados, los autores pueden ingresar a la plataforma de gestión editorial y verificar el estado de revisión, edición o publicación del artículo.

\section{A Canje}

La Revista de Arquitectura (Bogotá) está interesada en establecer canje con publicaciones académicas, profesionales o científicas del área de Arquitectura y Urbanismo, como medio de reconocimiento y discusión de la producción científica en el campo de acción de la publicación.

\section{Mecanismo}

Para establecer canje por favor descargar, diligenciar y enviar el formato: RevArq FP20 Canjes
Universidad Católica de Colombia (2020, enero-junio). Revista de Arquitectura (Bogotá), 22(I) I-188. Doi: 10.14718

ISSN: 1657-0308 E-ISSN: 2357-626X

Especificaciones:

Formato: $34 \times 24 \mathrm{~cm}$

Papel: Mate $115 \mathrm{~g}$

Tintas: Negro y policromía
A Contacto

Dirección postal:

Avenida Caracas No. 46-72.

Universidad Católica de Colombia

Bogotá D.C.(Colombia)

Código postal: 111311

Facultad de Diseño Centro de Investigaciones (CIFAR). Sede El Claustro. Bloque " $\mathrm{L}$ ", 4 piso Diag. 46A No. $15 \mathrm{~b}-10$ Editor, Arq. César Eligio-Triana

Teléfonos:

+57 (1) $3277300-3277333$

Ext. 3109; 3112 o 5146

Fax: +57 (1) 2858895
Correo electrónico: revistadearquitectura@ucatolica.edu.co cifar@ucatolica.edu.co

Página WEB: www.ucatolica.edu.co vínculo Revistas científicas

http://publicaciones.ucatolica.edu.co revistas-cientificas http://editorial.ucatolica.edu.co/ojsucatolica/revistas ucatolica/index.php/RevArq 
